National Aeronautics and Space Administration

\title{
Application of Human-Autonomy Teaming (HAT) Patterns to Reduced Crew Operations (RCO)
}

R. Jay Shively ${ }^{1}$, Summer L. Brandt ${ }^{2}$, Joel Lachter ${ }^{2}$, Mike Matessa ${ }^{3}$, Garrett Sadler ${ }^{4}$, and Henri Battiste ${ }^{4}$

1NASA Ames Research Center 2San Jose State University ${ }^{3}$ Rockwell Collins

${ }^{4}$ NVH Human Systems Integration

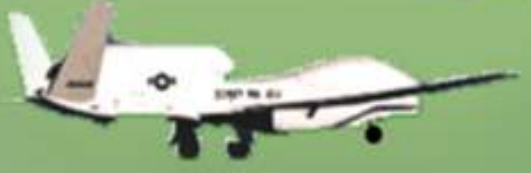




\section{Human-Autonomy Teaming}

Characteristics of HAT

- Bi-directional dialogue

- Shared goals

- Communication - shared language

- Trust

- Separate information that makes it sub-optimal to make decisions separately

- Shared (but not completely) Info/SA

- Flexibility/Robust/Resilient

- Shared fate ?

Critical Aspects of HAT

- Collaboration

- Pilot directed dynamic interface

- Contextually driven levels of automation and interaction 


\section{HAT Agent Architecture}

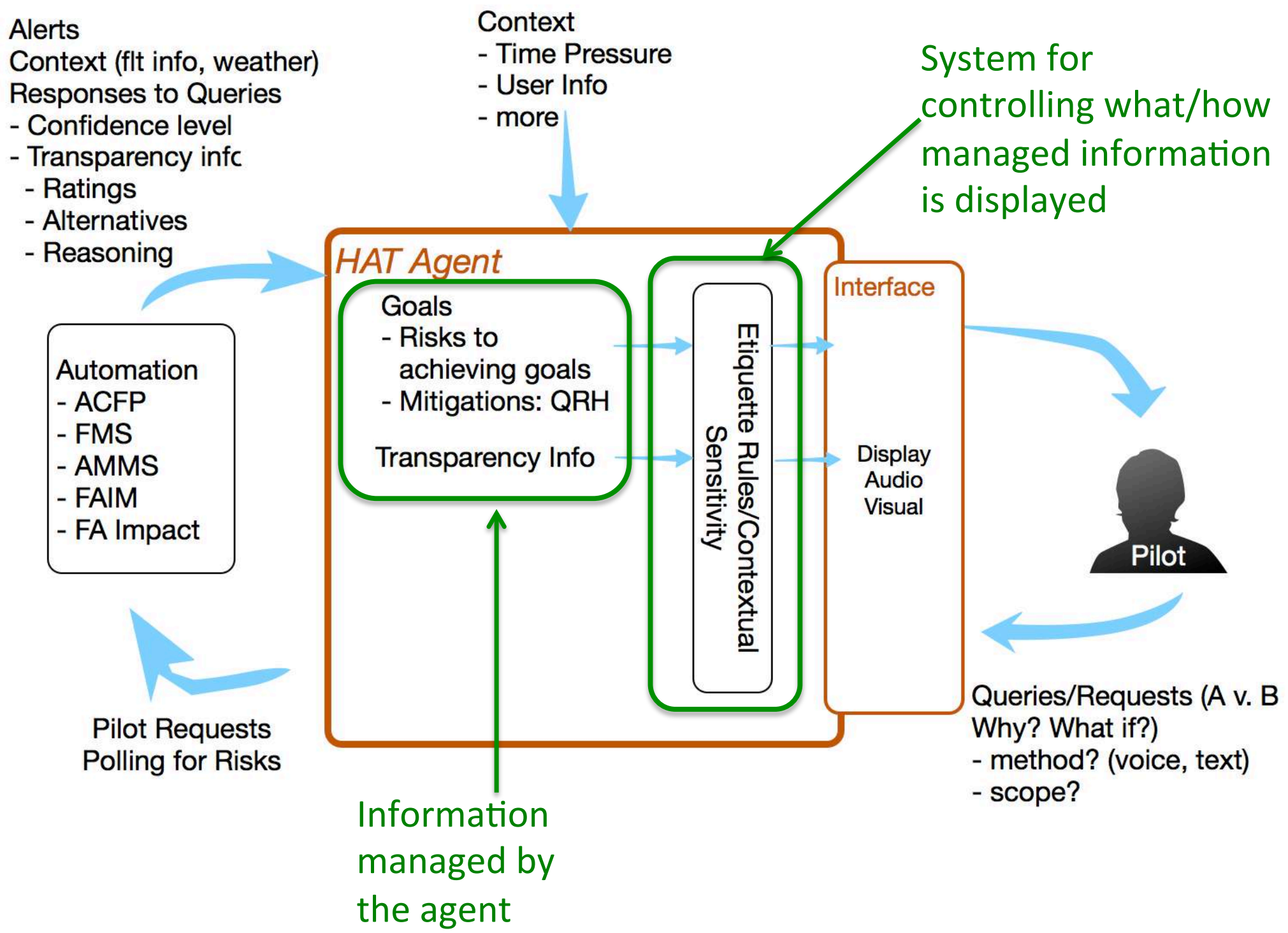


Human Operator

ㄹ. Intelligent / Cognitive Agent

Automated Tools

c........... Communication Only

Supervisory Relationship

Cooperative Relationship

Co-location (e.g., onboard an airplane, in ground station) 


\section{RCO Use-Case}

FLYSKY12 is en route from SFO to BOS. There is one POB and a dispatcher flight following.

- Onboard automation detects fuel imbalance and alerts POB and dispatcher.

- $P O B$ requests automation diagnose fuel imbalance. Automation reports to POB a leak in left tank.

- $\mathrm{POB}$ requests that agent manage fuel. Agent opens the cross feed and turns off the pumps in the right side to draw fuel from the left.

- POB contacts dispatch about need to divert.

- Dispatcher requests divert planning from dispatch automation.

- Dispatcher uplinks flight plan to POB. POB inspects the flight plan and agrees.

- POB requests agent coordinate divert with ATC. Agent reports divert is approved. POB tells agent to execute. 


\section{Dialog/Bi-Directional Communication Pattern}

Both the automation and human operators may have information or know of constraints the other is unaware of. Sharing this information is important to making plans that are acceptable and implementable

- Transparency

- In order to evaluate a recommendation, it is necessary to understand how that recommendation was arrived at

- Shared Language

- Human and computer reasoning systems often take very different forms (e.g., humans categorize and satisfice; computers are more quantitative). Dialog and Transparency require an interface that bridges such differences

- Human Directed

- Ultimate responsibility needs to fall somewhere. We believe that is going to be the human. It follows that the human should be giving explicit direction to the automation. 